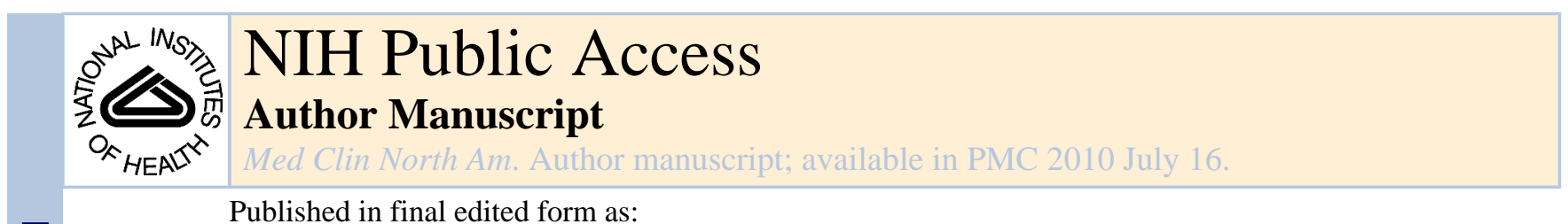

Published in final edited form as:

Med Clin North Am. 2008 January ; 92(1): 41-x. doi:10.1016/j.mcna.2007.09.005.

\title{
Genetics of Atrial Fibrillation
}

\author{
Patrick T. Ellinor ${ }^{1}$, B. Alexander $\mathbf{Y i}^{2}$, and Calum A. MacRae ${ }^{3}$ \\ ${ }^{1}$ Assistant Professor, Cardiac Arrhythmia Service and Cardiovascular Research Center, \\ Massachusetts General Hospital and Harvard Medical School, Boston, MA 02114 \\ ${ }^{2}$ Clinical and Research Fellow, Cardiology Division, Massachusetts General Hospital and Harvard \\ Medical School, Boston, MA 02114 \\ ${ }^{3}$ Assistant Professor, Cardiology Division and Cardiovascular Research Center, Massachusetts \\ General Hospital and Harvard Medical School, Boston, MA 02114
}

\section{Keywords}

Atrial fibrillation; arrhythmia; mutation; gene; genetics

\section{Introduction}

Atrial fibrillation (AF) is the most common cardiac arrhythmia. It affects over two million Americans, a number that will more than double by $2020^{1}$. The clinic visits, hospitalizations, medications, and procedures necessary to treat AF cost in excess of 6.4 billion dollars per year2. It accounts for one third of all strokes in patients above the age of 652 and is associated with an increased mortality $3,{ }^{4}$. While often associated with hypertension and structural heart disease, it is also seen in the setting of acute illness and in those who have undergone thoracic surgery. Traditionally, AF has not been considered a genetic condition; however, a number of recent studies have demonstrated that some forms of the arrhythmia, and in particular lone AF, have a substantial genetic basis ${ }^{5-8}$. Mutations in several ion channels have been identified in individuals and families with $\mathrm{AF}^{9-13}$, but appear to be rare causes of the arrhythmia ${ }^{14}, 15$. In the course of this review we will discuss the heritability of AF, the methods employed to identify the causal genes underlying an inherited disorder, and our current understanding of the specific genes implicated to date in AF.

\section{Atrial fibrillation is a heritable condition}

A genetic predisposition for AF has until recently not been well appreciated. It has long been reported that AF develops in some individuals at a relatively young age despite the absence of any evidence of structural heart disease and without any apparent etiology, though these families were typically considered rare ${ }^{16}$.

(C) 2007 Elsevier Inc. All rights reserved.

Corresponding author for proof and reprints: Patrick T. Ellinor, MD, PhD Cardiac Arrhythmia Service and Cardiovascular Research Center Massachusetts General Hospital 55 Fruit Street Boston, Massachusetts 02114 Phone: 617-724-8729 FAX: 617-726-2155 pellinor@partners.org Co-Authors: B. Alexander Yi Massachusetts General Hospital Cardiology Division 55 Fruit Street Bigelow 852 Boston, MA 02114 Calum A. MacRae Cardiovascular Research Center Massachusetts General Hospital 55 Fruit Street Boston, Massachusetts 02114.

Publisher's Disclaimer: This is a PDF file of an unedited manuscript that has been accepted for publication. As a service to our customers we are providing this early version of the manuscript. The manuscript will undergo copyediting, typesetting, and review of the resulting proof before it is published in its final citable form. Please note that during the production process errors may be discovered which could affect the content, and all legal disclaimers that apply to the journal pertain. 
In 2003, Fox and coworkers prospectively studied more than 5,000 individuals whose parents were enrolled in the original Framingham Heart Study ${ }^{7}$. Over a nineteen year follow up period, they found that AF in the offspring was independently associated with parental AF particularly if the subset was limited to those under the age of 75 and if those with antecedent heart disease were excluded. Having a parent with AF approximately doubled the four-year risk of developing AF even after adjustment for risk factors such as hypertension, diabetes mellitus, or myocardial infarction.

A genetic predisposition for $\mathrm{AF}$ in the general population was also suggested by a study of Icelanders by Arnar and colleagues in $2006^{5}$. After identifying more than 5,000 Icelanders with $\mathrm{AF}$ and then assessing relatedness from a nationwide genealogical database, eighty percent of those with AF were related within four meioses to another individual with AF. First-degree relatives of those with $\mathrm{AF}$ had a 1.77 higher relative risk for AF compared with the general population. The relative risk was 4.67 when the researchers only looked at patients under 60 years old.

The heritability of AF has also been examined in more selected patient populations. In a chart review of over two thousand patients referred to the arrhythmia clinic for AF, investigators at the Mayo Clinic found that 5\% had a family history of AF. This number was as high as $15 \%$ among patients with lone $\mathrm{AF}^{8}$. In 2005, in a study with pre-specified ascertainment, we found that nearly $40 \%$ of individuals with lone AF referred to the Arrhythmia Service at Massachusetts General Hospital had at least one relative with the arrhythmia, and a substantial number reported having multiple relatives with the arrhythmia ${ }^{6}$. In over $90 \%$ of cases, AF in the relatives could be verified. To obtain a crude index of heritability, we determined that prevalence of AF among each class of relative compared to the prevalence of age and sexmatched subjects. We found a significantly increased relative risk of AF among family members ranging from 2 fold in fathers to nearly 70 fold in male siblings.

\section{Genetic studies in atrial fibrillation}

Once a condition is found to be heritable, there are several techniques that are commonly employed to identify the genetic basis of a disease, namely, linkage analysis, candidate gene resequencing and association studies. We will discuss each of these methods in turn as they have been applied to AF.

Linkage analysis-The genes that underlie simple monogenic disorders with a Mendelian pattern of inheritance can be identified using linkage analysis. When passed from generation to generation, genetic markers that lie close together on the same chromosome are likely to be transmitted en bloc in proportion to their proximity to each other. A genome wide search for groups of markers that co-segregate with the disease as it travels through a family tree is performed to identify the approximate location of a genetic disease locus. Linkage studies report a logarithm of the odds or LOD score that reflects the likelihood of two markers or a marker and disease co-segregating when compared with chance alone. A LOD score of 3 or more (or odds of greater than a 1000:1) is considered to be statistically significant. Traditionally, restriction enzyme sites and microsatellite repeats have been used as genetic markers, but more recently, it has become possible to use single nucleotide polymorphisms or SNPs ${ }^{17}$. The ease of use in genotyping have made SNPs the most widely used genetic markers today.

Linkage analysis can be used to narrow the search for a causative gene to a chromosomal locus or relatively small region of the human genome associated with disease; however, this minimal genetic interval may still contain hundreds of genes spread over millions of base pairs. Once a genetic locus is identified, online data from human genome databases that have been developed as a direct result of the Human Genome Project are used to identify candidate genes 
within the genetic locus. These individual genes are then sequenced in affected individuals in an attempt to identify sequence variants that correlate with the disease. Once a base pair change is identified, it is then important to differentiate between a mutation and a genetic polymorphism or more common variant in the genome. For a sequence alteration to be considered a mutation it must segregate with the disease, have a plausible mechanism, and not be found in normal controls. Ultimately, the mutation should be sufficient to cause the phenotype, either in a human kindred or in a genetic model organism. There are several genetic loci that have been reported in large kindreds with Mendelian AF; on Chromosomes 5, 6, and 10 (see Table 1) ${ }^{9,18,19}$. In one such family of Chinese descent, Chen and coworkers identified a mutation in $\mathrm{KCNQ} 1^{9}$, a potassium channel that underlies the slow repolarizing current in cardiomyocytes known as $\mathrm{I}_{\mathrm{Ks}}$ (see Figure 1). From a four-generation family with AF, they were able to map the disease locus to a 12 megabase region on the short arm of chromosome 11. The KCNQ1 gene was located within this region and sequencing of the gene revealed a serine to glycine missense mutation at position 140 (S140G) in affected family members. The S140G mutation is located in the first transmembrane-spanning segment ${ }^{20}$ at the outer edges of the voltage-sensing domain and far from the pore-forming region of the potassium channel structure. Unlike the mutations in KCNQ1 associated with the long QT syndrome, which typically result in a loss of channel function, the S140G mutation resulted in a gain of channel function. In cultured cells, expression of the S140G mutant channel resulted in dramatically enhanced potassium channel currents and markedly altered potassium channel gating kinetics, changes that would be predicted to increase $\mathrm{I}_{\mathrm{Ks}}$. Such an increase would be expected to lead to a shortening of the action potential duration and thus make atrial myocytes vulnerable to reentry and subsequent $\mathrm{AF}$ (see Figure 1).

While the identification of this mutation provided an initial inroad into the pathogenesis of AF, this family also illustrates our limited understanding of the role of the KCNQ1 channel in atrial versus ventricular repolarization. Specifically, it remains unclear why with a mutation that results in an in vitro gain of function in KCNQ1, is associated with delayed ventricular repolarization (as manifest by a prolonged QT interval on their electrocardiograms) in more than half of the individuals with the S140G mutation exhibit.

Other gain of function mutations in KCNQ1 have been associated with the short QT syndrome $^{21}$. Hong and colleagues reported an unusual case of AF detected in utero and confirmed on electrocardiogram upon delivery of the newborn ${ }^{22}$. The baby's electrocardiogram also displayed a short QT interval. Based on this association, they sequenced the KCNQ1 gene and found a valine to methionine mutation in position 141 (adjacent to the mutated position found by Chen and colleagues). Like the S140G mutation, V141M mutant channels when expressed in vitro displayed markedly enhanced current density and altered gating kinetics.

Candidate gene studies-A candidate gene can be any gene that is hypothesized to cause a disease. In the case of linkage analysis, a gene may be considered a candidate gene based on its location within the region of interest, as well on the basis of any other information suggesting that the gene may play a role in the disease process in question.

Based on the work relating KCNQ1 to AF, investigators have considered other potassium channels as potential candidate genes for $\mathrm{AF}$ and begun screening these genes in cohorts with AF.

Otway and colleagues examined 50 kindreds with AF and amplified the genes for KCNQ1 and KCNE1-3, accessory subunits of KCNQ1 ${ }^{23}$. They found a single mutation in KCNQ1 in only one family - an arginine to cysteine change at amino acid position 14 (R14C) in KCNQ1. Unlike the S140G mutation discovered by Chen and colleagues, R14C had no significant effect on KCNQ1/KCNE1 current amplitudes in cultured cells at baseline; however, upon exposure 
to hypotonic solution, mutant channels exhibited a marked increase in currents compared to wild type channels. Interestingly, of those who carried the R14C mutation, only those with left atrial dilatation had AF leading the authors to propose a "two-hit" hypothesis of AF. They also identified a mutation in KCNE2 in two of the kindreds. Like the S140G mutation in KCNQ1, the mutation in KCNE2 (R27C) also dramatically increased the amplitude of $\mathrm{I}_{\mathrm{Ks}}{ }^{10}$.

Finally, other work has suggested that the relationship between potassium channels and AF extends beyond $\mathrm{I}_{\mathrm{Ks}}$. The work of Xia and colleagues may also implicate $\mathrm{KCNJ}$, an inward rectifier potassium channel that underlies the $I_{K 1}$ current, in $A^{11}$. In their work, a V93I mutation was found in all affected members in one kindred with familial AF. The V93I also lead to gain of function KCNJ2 channels, which increase potassium current amplitudes, but there is still an incomplete understanding of how an increase in the background current $\mathrm{I}_{\mathrm{K} 1}$ might lead to atrial arrhythmias.

Thus far, outside of the research described above, the significance of potassium channel mutations in AF is unknown. We have screened our cohort with lone atrial fibrillation for mutations in $\mathrm{KCNQ} 1, \mathrm{KCNJ} 2$, and $\mathrm{KCNE} 1-5$ and were unable to find any mutations in those genes ${ }^{14}$. These findings suggest that potassium channels are an uncommon cause of AF and there is much more to be learned about the diversity of molecular pathways that lead to this arrhythmia.

The genes encoding the connexins, gap-junction proteins that mediate the spread of action potentials between cardiac myocytes, have also been examined as potential candidates for AF. Prior work has shown that mice with null alleles of GJA5, the gene for connexin40, exhibit atrial reentrant arrhythmias ${ }^{24}$. From this work, Gollob and coworkers considered this gene as a potential candidate in individuals with idiopathic AF who underwent pulmonary vein isolation surgery ${ }^{13}$. An analysis of DNA isolated from their cardiac tissue showed that four of the fifteen subjects had mutations in GJA5 that markedly interfered with the electrical coupling between cells. In three of the patients, DNA isolated from their lymphocytes lacked the same mutation in GJA5 suggesting that the connexin40 mutations arose after fertilization, possibly during cardiac embryogenesis. One of the four individuals carried in the mutation in both cardiac tissue and in their lymphocytes arguing that, in this instance, the mutation was transmitted in the germline; however, more information about the transmission of AF in relatives of this individual was not available.

Association studies-Although traditional methods such as linkage analysis can be applied to families where the phenotype and pattern of inheritance are consistent with a monogenic disorder, the mode of transmission for AF is less clear. Association studies have been used in an attempt to identify the genetic basis of AF and other apparently complex traits. In an association study, the frequency of a single nucleotide polymorphism or SNP in individuals with a disease is compared to that in control populations. Over the past ten years, many case control association studies have been performed in subjects with AF. These studies have typically tested a small number of variants and have been directed at candidate genes previously believed to be involved in AF. Examples include genes in the renin-angiotensin system ${ }^{25,26}$, interleukins $^{27}$, signaling molecules ${ }^{28}$, gap junction proteins ${ }^{29}$ and ion channels ${ }^{30-32}$ (summarized in Table 2). Unfortunately, these studies have been limited by a low prior probability of any polymorphism truly being associated with AF. Further complicating these analyses are the small sample sizes, a lack of replication in distinct populations, as well as phenotypic and genetic heterogeneity.

In recent years, genome wide association studies (GWAS) have been made possible by advancements in genotyping technology that allow investigators to assay hundreds of thousands of SNPs spread over the entire human genome. The studies are typically done using 
a case control study design similar to that used in epidemiology 33 . Genome wide association studies attempt to identify novel genetic polymorphisms that are significantly more or less common in a group with a disease as compared to a control group. Since the markers are spread over the entire genome, these experiments are unbiased with no weight given to previously known candidate genes. Such studies have been used successfully in the past year to identify potential novel pathways for diabetes, macular degeneration, and repolarization.

While GWAS have the potential to identify new pathways for disease, they also have a number of limitations. In particular, with hundreds of thousands of individual associations being tested, these studies have a high likelihood of producing a false positive result. There is still discussion within the field of what the threshold $\mathrm{P}$ value should be for genome-wide significance ${ }^{34}$. False positive results can also emerge from population stratification or the failure to properly control for ethnicity thus resulting in over or under representation of spurious ethnic specific markers. While there have been proposed variations in study design in an effort to eliminate false associations, ultimately, replication of the results in other populations may be the best test of whether a result is a true positive ${ }^{33}$.

The biological significance of the identified variants is another concern. Most variants found in genetic association studies have been associated with relatively weak effects, e.g. relative risks on the order of $\sim 1.3$ to 1.5 . While these variants associated with a disease may generate new ideas about disease pathogenesis understanding the biological mechanism for most of these variants remains difficult.

Recently, a team led by the researchers at deCODE genetics have reported the results of a genome-wide association study for AF. Gudbjartsson and colleagues examined over 300,000 SNPs and identified two polymorphisms at a locus on the long arm of chromosome 4 (4q25) that demonstrated a highly significant association $\left(\mathrm{p}=3.3 \times 10^{-41}\right)$ with $\mathrm{AF}^{35}$. A strength of this work is that the investigators were able to replicate their original findings in other populations in Sweden, the United States and Hong Kong. Neither variant was correlated with obesity, hypertension, or myocardial infarction suggesting that the genetic variants are not associated with AF by affecting those risk factors.

How do the variants on chromosome 4 lead to AF? At present, the mechanism of action of these variants is unclear. Interestingly, these SNPs lie upstream from a gene that could plausibly play a role in the pathogenesis of AF: the paired-like homeodomain transcription factor 2, PITX2. This gene is known to play a role in the development of the left atrium ${ }^{36}$ and has been shown to be involved in suppression of pacemakers cells outside the sinus node in early development. Further work should help clarify the mechanism underlying the association of these markers with AF.

\section{Refining genetic studies of atrial fibrillation}

In order to continue to improve upon the utility of genetic studies for AF we will need to overcome a number of obstacles. A critical step in any genetic study is the ability to correctly assign the diagnosis. While on first pass this may seem straightforward; it can be challenging in $\mathrm{AF}$, a condition that can be asymptomatic, paroxysmal and have an onset later in life. Further complicating studies of AF are the genotypic and phenotypic heterogeneity. Rather than a single entity, AF may represent the final common pathway for a number of distinct pathogenic insults such as heart failure, hypertension, or thyroid abnormalities.

In order to address these challenges, we will have to continue to improve upon the characterization and classification of AF. The identification of endophenotypes or subtle, heritable traits that co-segregate with AF may help to refine ongoing genetic studies. For AF, endophenotypes such as specific $\mathrm{P}$ wave morphologies, pulmonary venous anatomy as assessed 
by computed tomography or magnetic resonance imaging, or biomarkers that are heritable and easily detectable may be helpful.

\section{Summary}

In summary, recent studies of AF have identified mutations in a series of ion channels; however, these channels appear to be relatively rare causes of AF. Recent genome wide association studies for AF have identified novel variants associated with the disease, though the mechanism of action for these variants remains unknown. Ultimately, a greater understanding of the genetics of AF should yield insights into novel pathways, therapeutic targets and diagnostic testing for this common arrhythmia.

\section{Acknowledgments}

This work was supported by NIH awards to Dr. Ellinor (HL71632) and Dr. MacRae (HL75431).

\section{References}

1. Go AS, Hylek EM, Phillips KA, et al. Prevalence of diagnosed AF in adults: national implications for rhythm management and stroke prevention: the AnTicoagulation and Risk Factors in AF (ATRIA) Study. JAMA May 9;2001 285(18):2370-5. [PubMed: 11343485]

2. Coyne KS, Paramore C, Grandy S, Mercader M, Reynolds M, Zimetbaum P. Assessing the direct costs of treating nonvalvular AF in the United States. Value Health Sep-Oct;2006 9(5):348-56. [PubMed: 16961553]

3. Benjamin EJ, Wolf PA, D'Agostino RB, Silbershatz H, Kannel WB, Levy D. Impact of AF on the risk of death: the Framingham Heart Study. Circulation Sep 8;1998 98(10):946-52. [PubMed: 9737513]

4. Gajewski J, Singer RB. Mortality in an insured population with AF. JAMA Apr 17;1981 245(15): 1540-4. [PubMed: 7206163]

5. Arnar DO, Thorvaldsson S, Manolio TA, et al. Familial aggregation of AF in Iceland. Eur Heart J Mar; 2006 27(6):708-12. [PubMed: 16428254]

6. Ellinor PT, Yoerger DM, Ruskin JN, MacRae CA. Familial aggregation in lone AF. Hum Genet Nov; 2005 118(2):179-84. [PubMed: 16133178]

7. Fox CS, Parise H, D'Agostino RB Sr. et al. Parental AF as a risk factor for AF in offspring. JAMA Jun 16;2004 291(23):2851-5. [PubMed: 15199036]

8. Darbar D, Herron KJ, Ballew JD, et al. Familial AF is a genetically heterogeneous disorder. J Am Coll Cardiol 2003;41(12):2185-92. [PubMed: 12821245]

9. Chen YH, Xu SJ, Bendahhou S, et al. KCNQ1 gain-of-function mutation in familial AF. Science Jan 10;2003 299(5604):251-4. [PubMed: 12522251]

10. Yang Y, Xia M, Jin Q, et al. Identification of a KCNE2 gain-of-function mutation in patients with familial AF. Am J Hum Genet Nov;2004 75(5):899-905. [PubMed: 15368194]

11. Xia M, Jin Q, Bendahhou S, et al. A Kir2.1 gain-of-function mutation underlies familial AF. Biochem Biophys Res Commun Jul 15;2005 332(4):1012-9. [PubMed: 15922306]

12. Olson TM, Alekseev AE, Liu XK, et al. Kv1.5 channelopathy due to KCNA5 loss-of-function mutation causes human AF. Hum Mol Genet Jul 15;2006 15(14):2185-91. [PubMed: 16772329]

13. Gollob MH, Jones DL, Krahn AD, et al. Somatic mutations in the connexin 40 gene (GJA5) in AF. N Engl J Med Jun 22;2006 354(25):2677-88. [PubMed: 16790700]

14. Ellinor PT, Moore RK, Patton KK, Ruskin JN, Pollak MR, Macrae CA. Mutations in the long QT gene, KCNQ1, are an uncommon cause of AF. Heart Dec;2004 90(12):1487-8. [PubMed: 15547041]

15. Ellinor PT, Petrov-Kondratov VI, Zakharova E, Nam EG, MacRae CA. Potassium channel gene mutations rarely cause AF. BMC Med Genet 2006;7:70. [PubMed: 16887036]

16. Wolff L. Familial auricular fibrillation. New Eng J Med 1943;229:396-8.

17. The International HapMap Consortium. A haplotype map of the human genome. Nature Oct 27;2005 437(7063):1299-320. [PubMed: 16255080] 
18. Brugada R, Tapscott T, Czernuszewicz GZ, et al. Identification of a genetic locus for familial AF. N Engl J Med Mar 27;1997 336(13):905-11. [PubMed: 9070470]

19. Ellinor PT, Shin JT, Moore RK, Yoerger DM, MacRae CA. Locus for AF maps to chromosome 6q14 -16. Circulation 2003;107(23):2880-3. [PubMed: 12782570]

20. Schenzer A, Friedrich T, Pusch M, et al. Molecular Determinants of KCNQ (Kv7) K+ Channel Sensitivity to the Anticonvulsatn Retigabine. Journal of Neuroscience 2005;25(20):5051-60. [PubMed: 15901787]

21. Bellocq C, van Ginneken ACG, Bezzina CR, et al. Mutation in the KCNQ1 Gene Leading to the Short QT-Interval Syndrome. Circulation 2004;109:2394-7. [PubMed: 15159330]

22. Hong K, Piper DR, Diaz-Valdecantos A, et al. De novo KCNQ1 mutation responsible for AF and short QT syndrome in utero. Cardiovasc Res Dec 1;2005 68(3):433-40. [PubMed: 16109388]

23. Otway R, Vandenberg JI, Guo G, et al. Stretch-sensitive KCNQ1 mutation: A link between genetic and environmental factors in the pathogenesis of AF? J Am Coll Cardiol 2007;49(5):578-86. [PubMed: 17276182]

24. Hagendorff A, Schumacher B, Kirchhoff S, Luderitz B, Willecke K. Conduction disturbances and increased atrial vulnerability in connexin40-deficient mice analyzed by transesophageal stimulation. Circulation 1999;99:1508-15. [PubMed: 10086977]

25. Tsai CT, Lai LP, Lin JL, et al. Renin-angiotensin system gene polymorphisms and AF. Circulation Apr 6;2004 109(13):1640-6. [PubMed: 15023884]

26. Bedi M, McNamara D, London B, Schwartzman D. Genetic susceptibility to AF in patients with congestive heart failure. Heart Rhythm 2006;3(7):808-12. [PubMed: 16818212]

27. Gaudino M, Andreotti F, Zamparelli R, et al. The $-174 \mathrm{G} / \mathrm{C}$ interleukin-6 polymorphism influences postoperative interleukin-6 levels and postoperative AF. Is AF an inflammatory complication? Circulation 2003;108(Suppl 1):II195-199. [PubMed: 12970232]

28. Schreieck J, Dostal S, von Beckerath N, et al. C825T polymorphism of the G-protein beta3 subunit gene and AF: association of the TT genotype with a reduced risk for AF. Am Heart J 2004;148(3): 545-50. [PubMed: 15389246]

29. Juang JM, Chern YR, Tsai CT, et al. The association of human connexin 40 genetic polymorphisms with AF. Int J Cardiol 2007;116(1):107-12. [PubMed: 16814413]

30. Lai LP, Su MJ, Yeh HM, et al. Association of the human minK gene $38 \mathrm{G}$ allele with AF: evidence of possible genetic control on the pathogenesis of AF. Am Heart J Sep;2002 144(3):485-90. [PubMed: 12228786]

31. Ravn LS, Hofman-Bang J, Dixen U, et al. Relation of 97T polymorphism in KCNE5 to risk of AF. Am J Cardiol Aug 1;2005 96(3):405-7. [PubMed: 16054468]

32. Zeng Z, Tan C, Teng S, et al. The Single Nucleotide Polymorphisms of I(Ks) Potassium Channel Genes and Their Association with AF in a Chinese Population. Cardiology Sep 29;2006 108(2):97103. [PubMed: 17016049]

33. Risch NJ. Searching for genetic determinants in the new millennium. Nature Jun 15;2000 405(6788): 847-56. [PubMed: 10866211]

34. Hunter DJ, Kraft P. Drinking from the Fire Hose--Statistical Issues in Genomewide Association Studies. New England Journal of Medicine 2007;357(5):436-9. [PubMed: 17634446]

35. Gudbjartsson DF, Arnar DO, Helgadottir A, et al. Variants conferring risk of AF on chromosome 4q25. Nature 2007;448(7151):353-7. [PubMed: 17603472]

36. Franco D, Campione M. The role of Pitx2 during cardiac development. Linking left-right signaing and congenital heart diseases. Trends Cardiovasc. Med 2003;13:157-63. [PubMed: 12732450]

37. Zini S, Fournie-Zaluski MC, Chauvel E, Roques BP, Corvol P, Llorens-Cortes C. Identification of metabolic pathways of brain angiotensin II and III using specific aminopeptidase inhibitors: predominant role of angiotensin III in the control of vasopressin release. Proc. Natl. Acad. Sci, USA 1996;93:11968-73. [PubMed: 8876246]

38. Ellinor PT, MacRae CA. The Genetics of AF. J Cardiovasc Electrophysiol September;2003 14:10079. 2003. [PubMed: 12950549]

39. Wilde AAM, Bezzina CR. Genetics of cardiac arrhythmias. Heart 2005;91:1352-8. [PubMed: 16162633] 
40. Oberti C, Wang L, Li L, et al. Genome-wide linkage scan identifies a novel genetic locus on chromosome $5 \mathrm{p} 13$ for neonatal AF associated with sudden death and variable cardiomyopathy. Circulation Dec 21;2004 110(25):3753-9. [PubMed: 15596564]

41. Volders PG, Zhu Q, Timmermans C, et al. Mapping a novel locus for familial AF on chromosome 10p11-q21. Heart Rhythm Apr;2007 4(4):469-75. [PubMed: 17399636]

42. Asselbergs FW, Moore JH, van den Berg MP, et al. A role for CETP TaqIB polymorphism in determining susceptibility to AF: a nested case control study. BMC Med Genet 2006;7:39. [PubMed: 16623947] 


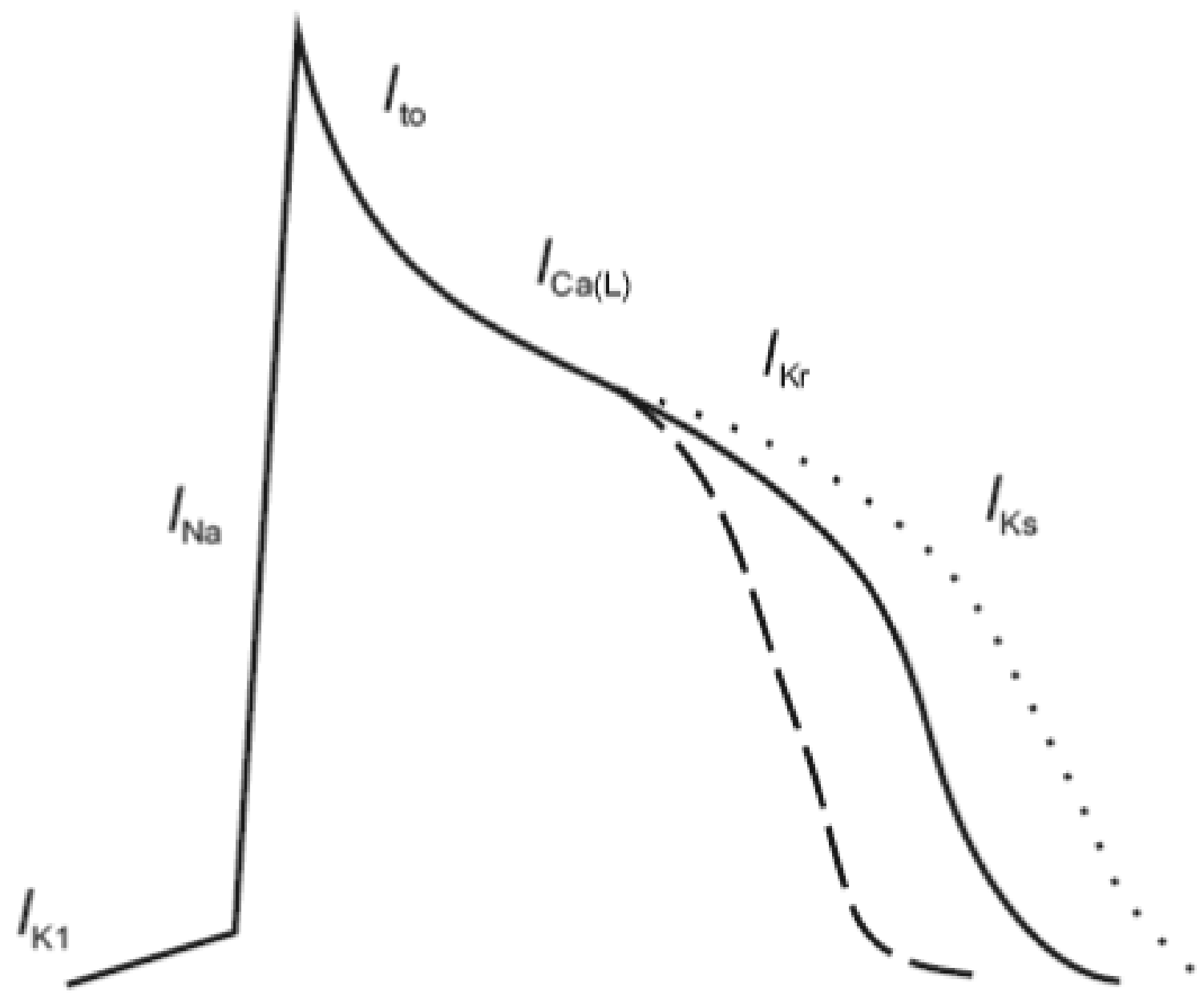

Figure 1.

Both gain of function and loss of function mutations in $\mathrm{I}_{\mathrm{Ks}}$ have been associated with AF. Mutations in KCNQ1 and KCNE2 increase the current $\mathrm{I}_{\mathrm{Ks}}$, which is predicted to shorten the action potential (dashed line) in cardiac myocytes and render atrial myocytes susceptible to reentrant arrhythmias. Mutations in KCNA5 (Kv1.5) that are predicted to prolong the action potential duration (dotted line) have also been associated with AF. 
